\title{
Single Rotational Product Propensity in the Photodissociation of HOD
}

\author{
S. A. Harich, X. F. Yang, and X. Yang* \\ Institute of Atomic and Molecular Sciences, Academia Sinica, Taipei, Taiwan, Republic of China \\ R. van Harrevelt and M. C. van Hemert \\ Leiden Institute of Chemistry, Gorlaeus Laboratories, Leiden University, P.O. Box 9502, 2300 RA Leiden, The Netherlands \\ (Received 21 February 2001; published 4 December 2001)
}

\begin{abstract}
Photodissociation of HOD from the $\tilde{\mathrm{B}}$ state has been studied using the high resolution rydberg "tagging" time-of-flight (TOF) technique. The TOF spectra show an unusually strong population $(\approx 50 \%)$ for a single rotational state for the $\operatorname{OD}\left(A^{2} \Sigma, v=0\right)$ fragments. Through theoretical studies, this phenomenon, which we have labeled the "single rotational product propensity," is attributed to a dynamically constrained threshold effect in the photodissociation of the HOD molecule on the $\tilde{\mathrm{B}}$ state.
\end{abstract}

DOI: 10.1103/PhysRevLett.87.263001

Photodissociation of water molecules in the vacuum ultraviolet region has been a prototype system for unimolecular dissociation, because this is one of the few photodissociation processes that can be studied fully theoretically from first principles [1]. It is also an important process in the upper terrestrial atmosphere, in cometary and planetary atmospheres, and in diffuse interstellar clouds. In this Letter, we focus on the dynamics after excitation into the electronically excited $\tilde{\mathrm{B}}$ state with Lyman $\alpha(121.6 \mathrm{~nm})$ radiation, which is abundant in stellar radiation. Direct dissociation on this surface leads to electronically excited $\mathrm{OH}(A)$ fragments. Electronically nonadiabatic couplings between the $\tilde{\mathrm{B}}$ and $\tilde{\mathrm{X}}$ states are responsible for the dominant ground state $\mathrm{OH}$ channel. The general behavior of the rotational distribution for bent-linear transitions such as $\operatorname{HOD}(\tilde{\mathrm{B}}) \leftarrow \operatorname{HOD}(\tilde{\mathrm{X}})$ is well understood [2]. For direct dissociation, the rotational distribution is a smooth function of the rotational quantum number $N$, and is strongly inverted, which means that the distribution has a maximum at $N \gg 0$. The dynamics on the $\tilde{\mathrm{B}}$ state surface of water is not direct, as is evident from the oscillatory structure in the $\tilde{\mathrm{B}}$ band absorption spectrum. It is generally understood that this gives rise to interference between different pathways to the same rotational product state, resulting in a complicated structure in the rotational distributions $[3,4]$. These structures are lost in the usual room temperature experiments [5] due to averaging over the rotational levels of the parent molecule.

This paper describes experimental and theoretical evidence for a highly unusual rotational distribution for the $\operatorname{OD}(A, v=0)$ fragments formed after excitation into the $\tilde{\mathrm{B}}$ state with Lyman $\alpha$ photons $(121.6 \mathrm{~nm})$. Rotational distributions are measured using the rydberg tagging time-of-flight (TOF) method, and are calculated $a b$ initio using a three-dimensional time-dependent wave packet method. A strong population of about $50 \%$ was found for a single rotational level, $N=28$. This strong propensity for one single rotational product has never been found before in photodissociation studies, to the best of our knowledge. We label this phenomenon the
PACS numbers: $33.80 . \mathrm{G} \mathrm{j}$

"single rotational product propensity," or, briefly, the "single $N$ propensity". The rotational distributions for $\mathrm{H}_{2} \mathrm{O} / \mathrm{D}_{2} \mathrm{O}$ photodissociation found in recent experiments [4] similar to that described in this paper do not show the single $N$ propensity, but were in agreement with the conventional type of rotational distributions. The TOF technique used in this work was pioneered by Welge et al. [6,7]. In this method, the kinetic energy of the atomic hydrogen fragments is measured. The internal (electronic, vibrational, or rotational) excitation of the OD fragments then follows from conservation of momentum and energy. The experimental method used here has been described in detail previously in Ref. [4].

The time resolution in the TOF measurement is about $0.3 \%$. In order to study the HOD photodissociation, a

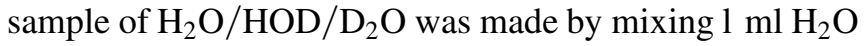
and $1 \mathrm{ml} \mathrm{D}_{2} \mathrm{O}$. The molecular beam was generated by bubbling Ar through this mixed water sample and then expanding the $\mathrm{Ar} /$ water mixture through a pulsed nozzle with a $0.5 \mathrm{~mm}$ diameter orifice. The detection axis, the molecular beam, and the photolysis laser beam are all perpendicular to each other in this experiment. Since the HOD sample used in the experiment contains $\mathrm{H}_{2} \mathrm{O}$ and $\mathrm{D}_{2} \mathrm{O}$, the TOF measurements of the $\mathrm{H}$ products from the mixed sample will have contributions from $\mathrm{H}_{2} \mathrm{O}$, while TOF measurements of the $\mathrm{D}$ products from the mixed sample will have contributions from $\mathrm{D}_{2} \mathrm{O}$. In order to subtract the contributions of $\mathrm{H}_{2} \mathrm{O}$ and $\mathrm{D}_{2} \mathrm{O}$, TOF measurements on the $\mathrm{H}$ and $\mathrm{D}$ products from $\mathrm{H}_{2} \mathrm{O}$ and $\mathrm{D}_{2} \mathrm{O}$, respectively, have also been done under similar experimental conditions. The polarization of the $121.6 \mathrm{~nm}$ laser light can be changed by rotating the polarization of the $845 \mathrm{~nm}$ laser light using a rotatable half-wave plate.

TOF spectra of the $\mathrm{H}$ atom products were obtained from the photodissociation of $\mathrm{H}_{2} \mathrm{O} / \mathrm{HOD} / \mathrm{D}_{2} \mathrm{O}$ and $\mathrm{H}_{2} \mathrm{O}$ using the experimental technique described above. Contributions from $\mathrm{H}_{2} \mathrm{O}$ were then subtracted from the results of the mixed sample. The experimental TOF spectra of the $\mathrm{H}$ atom from HOD were then converted into translational energy spectra in the center-of-mass frame. The upper part 
of Fig. 1 shows the translational energy spectra of the $\mathrm{H}$ atom products at $121.6 \mathrm{~nm}$ excitation using two different polarization schemes (parallel and perpendicular to the detection axis), for an energy up to $5000 \mathrm{~cm}^{-1}$. The spectra were taken at a total stagnation pressure of 600 torr of a sample of $3 \% \mathrm{H}_{2} \mathrm{O} / \mathrm{HOD} / \mathrm{D}_{2} \mathrm{O}$ in Ar. The rotational temperature of the water molecule at this expansion condition is estimated to be about $10 \mathrm{mK}$.

Since translational energy distributions for two detection schemes (parallel and perpendicular) were measured with a check at the magic angle, the anisotropy distribution for the dissociation process $\beta(E)$ and the total translational energy distribution $\phi(E)$ can be determined from Eq. (1):

$$
f(E, \theta) \approx \phi(E)\left[1+\beta(E) P_{2}(\cos \theta)\right],
$$

where $\theta=0^{\circ}$ for the parallel detection scheme, and $\theta=$ $90^{\circ}$ for the perpendicular detection scheme. Once $\beta(E)$ and $\phi(E)$ are known, the angular dependent translational energy distribution $f(E, \theta)$ can be determined: The result is shown in the lower part of Fig. 1, for an energy up to $5000 \mathrm{~cm}^{-1}$. Since the total energy must be conserved in the dissociation process HOD $+h \nu \rightarrow \mathrm{H}+\mathrm{OD}$, the
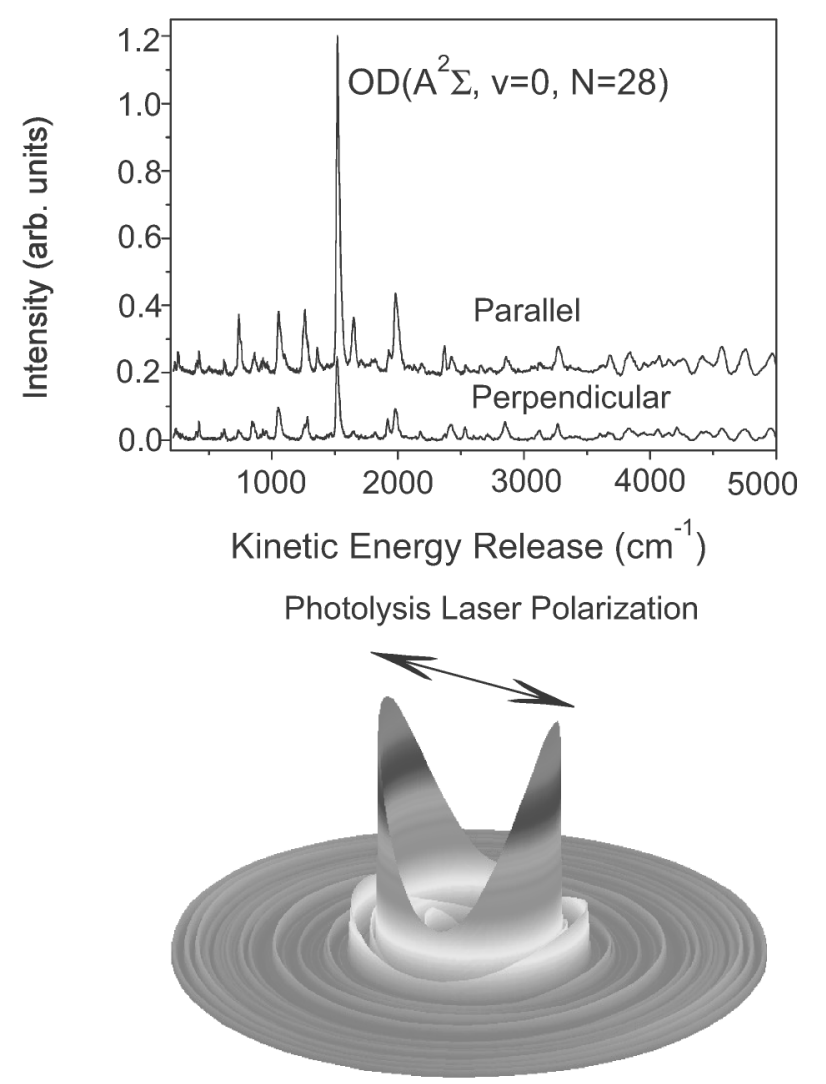

FIG. 1. Upper part: Experimental $\mathrm{H}$ atom translational kinetic energy distribution for the photodissociation of HOD at $121.6 \mathrm{~nm}$, with photolysis laser polarization parallel or perpendicular to the detection axis. Lower part: 3D angular dependent translational kinetic energy distribution, up to an energy of $5000 \mathrm{~cm}^{-1}$.
OD quantum state products from the photodissociation can be determined from the $\mathrm{H}$ atom translational energy distribution.

Based on previous spectroscopic data on the OD molecule, all sharp features in the translational energy spectra shown in Fig. 1 can be clearly assigned. The sharp structures in Fig. 1 are mostly due to the OD products in the electronically excited $A^{2} \Sigma$ state. Most of the $\operatorname{OD}\left(A^{2} \Sigma\right)$ products are in the $v=0$ state. The rotational state distribution of the $\mathrm{OD}\left(A^{2} \Sigma, v=0\right)$ products is shown in Fig. 2. The population of $N=28$ is much larger than the population of any other rotational state. This phenomenon, labeled the single $N$ propensity by us, was not observed in the photodissociation of similar systems such as $\mathrm{H}_{2} \mathrm{O}, \mathrm{D}_{2} \mathrm{O}$, and HOD with D detection at $121.6 \mathrm{~m}$. It seems that the observation of the single $N$ propensity is truly accidental for the HOD system at the photolysis energy (H Lyman $\alpha$ wavelength). Note that the single $N$ propensity is found here only for the parallel detection scheme. The structure in the distributions at lower $N$ values with amplitudes less than 5 times the single $N$ peak is often found in rotational distributions, and is related to interference.

The key to the understanding of the observed rotational distribution is the remarkable shape of the potential energy surface of the $\tilde{\mathrm{B}}$ state. Figure 3 shows a contour plot of the potential energy surface for a fixed OD bond length of 2.04 bohrs, which is equal to the equilibrium bond length of an OD molecule with $v=0$ and $N=28$, and is close to the optimal OD distance ( $\approx 2$ bohrs) everywhere on the potential energy surface. The surface has deep wells, about $2.5 \mathrm{eV}$ deep, at the two collinear geometries (HDO and $\mathrm{DOH})$. The initial position of the hydrogen atom is indicated with the cross in Fig. 3. Although the potential energy surface is repulsive in this region, the strong angular anisotropy makes the force point almost perpendicular to the radial direction. The hydrogen atom moves towards the linear DOH geometry, and gets large amount of orbital angular momentum. At the same time, the OD molecule

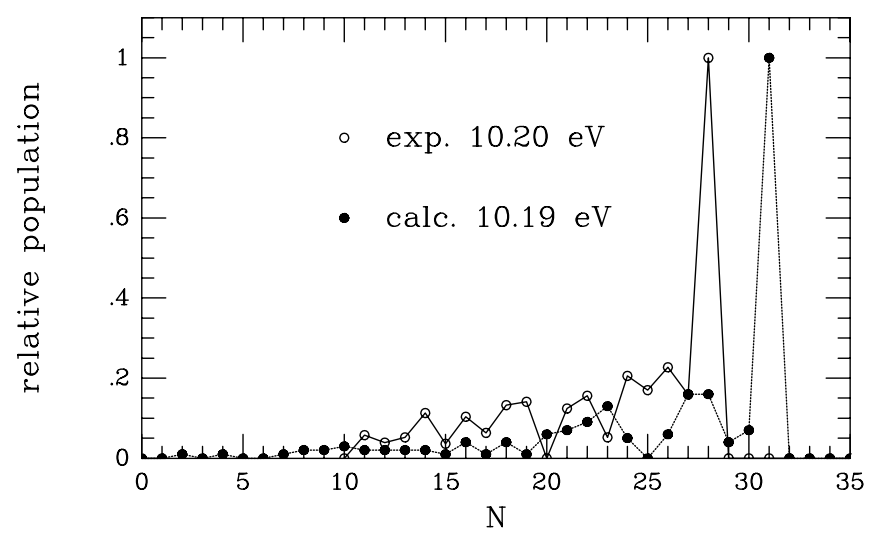

FIG. 2. Rotational population distributions, relative to the population of the dominant $N$ product, as obtained experimentally for a photon energy $E$ of $10.20 \mathrm{eV}$ (open circles), and from the calculations (closed circles) for $E=10.19 \mathrm{eV}$. 


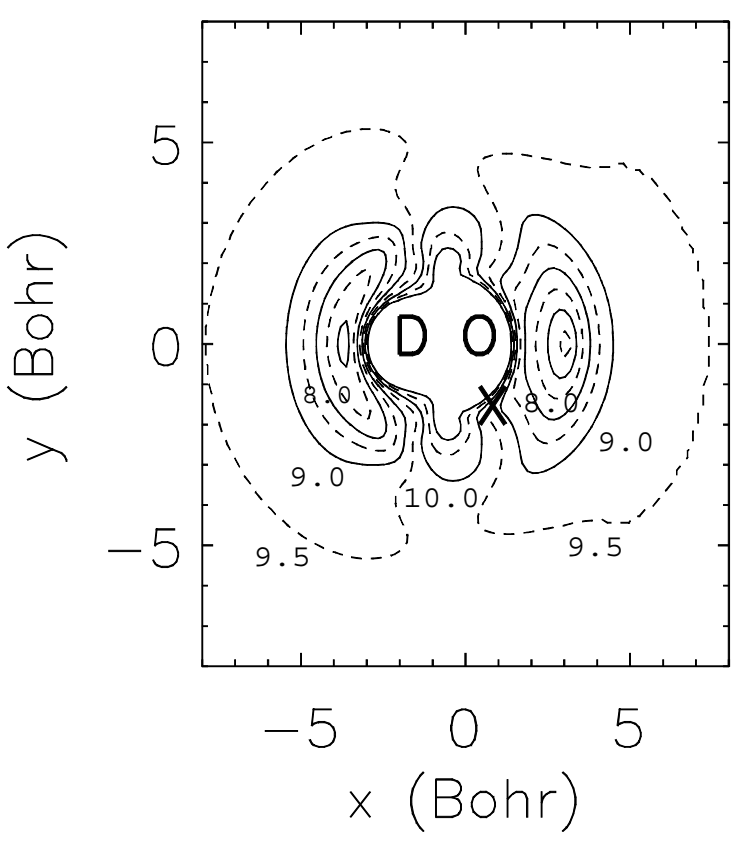

FIG. 3. Contour plot of the B state potential energy surface of Ref. [8], for an OD distance of 2.04 bohrs. The oxygen atom is located at $(x, y)=(0,0)$, the deuterium atom at $(-2.04,0)$. Contour lines are for $6.5,7,7.5, \ldots, 9.5$, and $10 \mathrm{eV}$. The initial location of $\mathrm{H}$ is indicated with the cross.

rotates clockwise in order to compensate the change in angular momentum of the hydrogen atom, since the total angular momentum must be conserved. When $N$ gets large, rotational barriers due to the $N(N+1) /\left(2 \mu_{R} R^{2}\right)$ term in the Hamiltonian are important. $R$ is the photofragmentation recoil distance, and $\mu_{R}$ is the reduced mass of the OD-H complex.

We have calculated the rotational barrier energies by diagonalizing the two-dimensional Hamiltonian for fixed $R$ values, using the $a b$ initio three-dimensional potential energy surface described in Ref. [8]. The eigenvalues form the set of adiabatic potentials $V_{v N}(R) ; V_{v N}(\infty)$ is equal to the energy of $\mathrm{OD}(v, N)+\mathrm{H}$. In Fig. 4 , the ab initio adiabatic potentials relative to the $\mathrm{OH}(A, v=0)$ threshold energy are shown for $v=0$ and for $N$ values between 27 and 33. The spectroscopic excess energy above the $\mathrm{OH}(A, v=0)$ threshold for the experimental wavelength $(121.6 \mathrm{~nm})(1.05 \mathrm{eV})$ is indicated with the dotted line in Fig. 4. States with $N \leq 31$ are open channels for the experimental wavelength, but for states with $28<N \leq 31$ the rotational barriers prevent direct dissociation, which explains the observation of the very small population of $N>28$ rotational levels. $N=28$ is the highest rotational state for which direct dissociation is possible and for which there are no quasibound levels resonant with the experimental energy. The latter implies that the single $N$ propensity is not related to excitation into a quasibound resonance state.

We have performed three-dimensional wave packet calculations, using the nuclear Hamiltonian for zero total nuclear angular momentum of the HOD system $\left(J_{\mathrm{HOD}}=0\right)$.

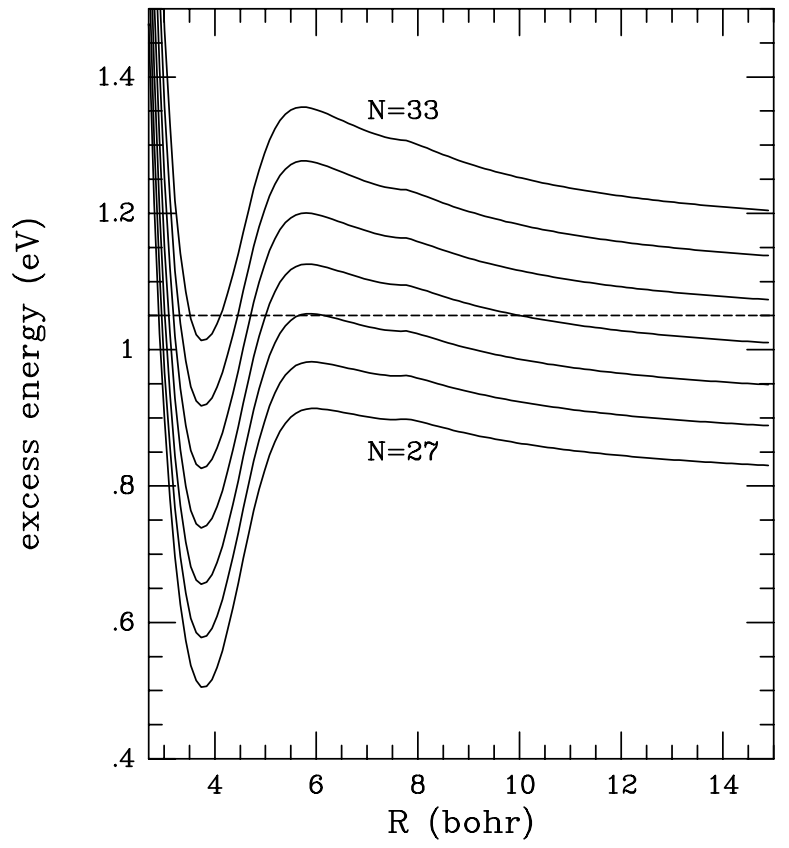

FIG. 4. Adiabatic potential energy curves $V_{v N}(R)$ for $v=0$ and $N$ between 27 and 33, obtained by diagonalizing the threedimensional Hamiltonian for fixed $R$ values. The excess energy is relative to the $\mathrm{OH}(A, v=0)$ threshold energy. The vertical broken line corresponds to the energy used in the experiment.

The wave packet calculations yield a set of $P_{N}(E)$ functions, the probability that the $\operatorname{OD}(A, v=0)$ fragments have a rotational quantum number $N$, at a photon energy $E$. The details of the calculations are similar as in Ref. [9]. For the present problem, a larger grid in the $R$ coordinate than before was necessary: A grid of 144 points between 1 and 15 bohrs was found to be adequate for sufficiently converged results. In the $a b$ initio surface for the $\tilde{\mathrm{B}}$ state, the $\mathrm{OH}(A, v=0)$ threshold is $0.19 \mathrm{eV}$ lower than the spectroscopic threshold. We therefore expect that in the calculations the single $N$ propensity occurs at an $N$ value of 31 instead of 28 at the same photon energy region as in the experiment $(10.2 \mathrm{eV})$.

In Fig. 5 are shown the $P_{N}(E)$ functions for $N=30$, 31 , and 32. The calculated barrier energies are indicated with the vertical dotted lines. Figure 5 clearly shows that the onsets of the $P_{N}(E)$ functions are close to the adiabatic barrier energies. For each of the three $N$ values, the $P_{N}$ function reaches quite high values $(\approx 0.5)$ at various energies close to the barrier energy. At each of these energies, the rotational distribution shows the single $N$ propensity. The maximum probability for $N=31$ is found at a photon energy of $10.19 \mathrm{eV}$. In Fig. 2 the calculated rotational distribution at this energy is presented. The theoretical rotational distributions resemble the experimental one. The $P_{N}(E)$ functions clearly show interference patterns typical for multiple photodissociation pathways. This interference is not responsible for the occurrence of the single $N$ propensity, which follows from the success of quasiclassical trajectory calculations to reproduce the single $N$ propensity [10]. 


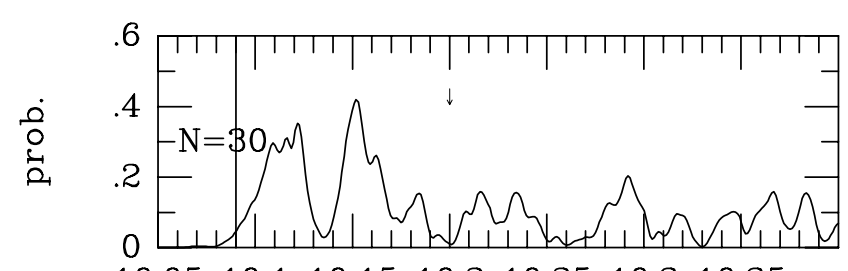

$\begin{array}{lllllll}10.05 & 10.1 & 10.15 & 10.2 & 10.25 & 10.3 & 10.35\end{array}$
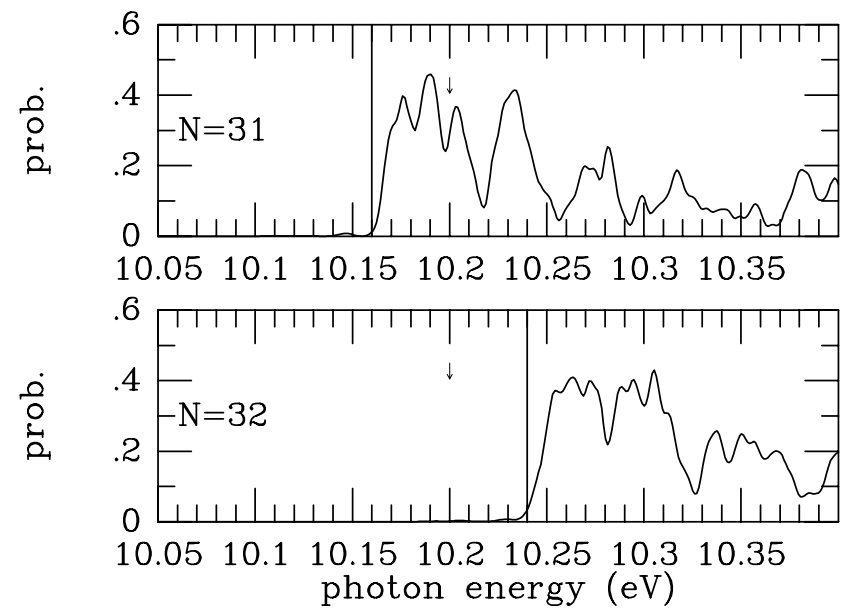

FIG. 5. Calculated probabilities of the $\operatorname{OD}(A, v=0)$ fragment to have a rotational quantum number $N\left(P_{N}\right)$, as a function of the photon energy, as obtained from three-dimensional quantum wave packet calculations, for $N=30,31$, and 32. The calculated barrier energies are indicated with the vertical dotted lines. The arrows indicate the experimental photon energy of $10.2 \mathrm{eV}$.

Essential to the single $N$ propensity is the sharp rise of the $P_{N}$ functions above the barriers. This is explained by the large torque at the initial position of the hydrogen atom. At the moment when the hydrogen atom attempts to escape, the rotational barrier can be too large because of the high value of $N$, and the $\mathrm{H}$ atom returns to the OD molecule. The hydrogen atom then attempts several times to escape the OD molecule, and during each attempt the torque generates high angular momenta for the OD and $\mathrm{H}$ motion, until finally $N$ is low enough for the $\mathrm{H}$ atom to overcome the barrier. This process leads to a strong preference for the formation of OD fragments with the highest possible $N$ value for which the barrier is low enough.

The single $N$ propensity is strongly energy dependent. For example, the calculated single $N$ propensity at $10.19 \mathrm{eV}$ disappears when the energy is $0.03 \mathrm{eV}$ higher or lower. The observation that the single $N$ phenomenon occurs only in narrow energy ranges explains why similar rotational distributions have not been found for the $\mathrm{OH}$ fragments formed during the photodissociation of $\mathrm{H}_{2} \mathrm{O}$ and HOD, and the OD fragments from $\mathrm{D}_{2} \mathrm{O}$ : The energies at which the single $N$ propensity occurs are different for each of the photodissociation variants. For the OD fragments of HOD, one of the energy regions, where the remarkable rotational distribution occurs, just happens to include Lyman alpha $(10.20 \mathrm{eV})$. Indeed, theoretical calculations on $\mathrm{H}_{2} \mathrm{O}$ and $\mathrm{D}_{2} \mathrm{O}$ yield similar $P_{N}$ functions as for $\mathrm{HOD} \rightarrow \mathrm{OD}+\mathrm{H}$ in the energy region around Lyman $\alpha$.

In the experiment, the single $N$ propensity was found only in the parallel direction. Since the theoretical approach followed in this paper does not allow to calculate orientation-dependent rotational distributions, this aspect requires further theoretical analysis. A possible explanation is given below. For direct dissociation leading to the single $N$ products, the recoil velocity vector is probably almost parallel to the transition dipole moment vector. In contrast, indirect dissociation pathways leading to low $N$ values are expected to result in a more isotropic distribution of the recoil direction. Thus, the dependence of the single $N$ propensity on the direction of observation suggests that pathways to the single $N$ rotational state are mainly direct.

In summary, both the experiment and calculations show rotational distributions strongly peaked at one single $N$ value. The calculations suggest that the single $N$ propensity is strongly energy dependent, and that, at energies slightly different from Lyman alpha energy, it may also be observed for photodissociation of $\mathrm{H}_{2} \mathrm{O}$ and $\mathrm{D}_{2} \mathrm{O}$. These theoretical findings can be tested if experiments with a tunable photolysis light become possible. It is expected that similar rotational distributions may be observed for photodissociation of other molecules, provided that the potential energy surface is strongly anisotropic at the equilibrium geometry of the ground state, and provided that the excitation energy is not too high compared to the dissociation energy.

*Also at the Department of Chemistry, National Tsing Hua University, Hsin-Chu, Taiwan, Republic of China.

[1] R. Schinke, Photodissociation Dynamics (Cambridge University, Cambridge, England, 1993).

[2] R. Schinke, J. Chem. Phys. 85, 5049 (1986).

[3] R. N. Dixon, D. W. Hwang, X. F. Yang, S. Harich, J. J. Lin, and X. Yang, Science 285, 1249 (1999).

[4] S. A. Harich, D. W. H. Hwang, X. F. Yang, J. J. Lin, X. M. Yang, and R. N. Dixon, J. Chem. Phys. 113, 10073 (2000).

[5] A. H. Zanganeh, J. H. Fillion, J. Ruiz, M. Castillejo, J. L. Lemaire, N. Shafizadeh, and F. Rostas, J. Chem. Phys. 112, 5660 (2000).

[6] J. Biesner, L. Schnieder, G. Ahlers, X. X. Xie, K. H. Welge, M. N. R. Ashfold, and R. N. Dixon, J. Chem. Phys. 91, 2901 (1989), and references therein.

[7] L. Schnieder, K. Seekamp-Rahn, E. Wrede, and K. H. Welge, J. Chem. Phys. 107, 6175 (1997).

[8] R. van Harrevelt and M. C. van Hemert, J. Chem. Phys. 112, 5777 (2000).

[9] R. van Harrevelt and M. C. van Hemert, J. Chem. Phys. 112, 5787 (2000).

[10] R. van Harrevelt, M. C. van Hemert, and G. C. Schatz, J. Phys. Chem. A (to be published). 